\title{
OPEN Observational learning of fear in real time procedure
}

\author{
Michał Szczepanik ${ }^{1,2,5}$, Anna M. Kaźmierowska ${ }^{1,2,5}$, Jarosław M. Michałowski ${ }^{3}$, \\ Marek Wypych ${ }^{1}$, Andreas Olsson ${ }^{4} \&$ Ewelina Knapska ${ }^{2 \bowtie}$
}

Learning to avoid threats often occurs by observing others. Most previous research on observational fear learning (OFL) in humans has used pre-recorded standardized video of an actor and thus lacked ecological validity. Here, we aimed to enhance ecological validity of the OFL by engaging participants in a real-time observational procedure (35 pairs of healthy male friends, age 18-27). One of the participants watched the other undergo a differential fear conditioning task, in which a conditioned stimulus (CS+) was paired with an aversive electric shock and another stimulus (CS-) was always safe. Subsequently, the CS+ and CS- were presented to the observer to test the OFL. While the friend's reactions to the shock elicited strong skin conductance responses (SCR) in all observers, subsequent differential SCRs (CS+ > CS-) were found only when declarative knowledge of the CS+/US contingency (rated by the participants) was acquired. Contingency-aware observers also showed elevated fear potentiated startle responses during both CS+ and CS- compared to baseline. We conclude that our real-time procedure can be effectively used to study OFL. The procedure allowed for dissecting two components of the OFL: an automatic emotional reaction to the response of the demonstrator and learning about stimulus contingency.

Learning through interactions with others, that is, social learning, is often adaptive. For instance, receiving information about threats through social means helps to avoid costly first-hand experiences. Social transfer of information about threats is thought to be mediated by emotional contagion, a bottom-up process through which the emotional state of one organism elicits the same state in another ${ }^{1}$. This basic ability to perceive and mimic the emotions of others is conserved across species, including apes ${ }^{2}, \operatorname{dogs}^{3}$ and birds ${ }^{4}$. Further, the ability to not only imitate emotions but also learn from emotional expressions of others has been described in different species, such as rhesus monkeys ${ }^{5}$ and rodents ${ }^{6,7}$. In humans, it has been shown that watching an actor undergoing fear conditioning evokes vicarious reactions in the observer resulting in learning as measured by behavioral, psychophysiological, and neural responses ${ }^{8,9}$ (observational fear learning, OFL). These responses can be modulated by a number of factors, including social group affiliation ${ }^{10}$, and racial similarity to the actor ${ }^{11}$. The role of empathy level of the observer in the OFL is not clear. It has been shown either to modulate ${ }^{12}$ or to have no effect on the OFL ${ }^{13}$.

In previous studies on vicarious fear learning in humans, participants observed an actor performing a differential fear conditioning task. As most of these studies have used standardized video recordings of an anonymous person presented to individual participants ${ }^{8,12}$ (with the exception of one recent experiment ${ }^{14}$ involving pairs $^{2}$ of participants unfamiliar to each other), they have lacked ecological validity offered by real-time interactions.

In this study, we modified the experimental protocol proposed by Haaker and colleagues ${ }^{15}$ to enhance its ecological validity and to test whether the modifications affected acquisition of fear. Ecologically valid (naturalistic) paradigms can be understood as involving realistic, interactive stimuli representative of real-world experiences while maintaining a reasonable degree of experimental control ${ }^{16,17}$. We believe that modifying the procedure toward a more naturalistic one can tell us how OFL occurs when authentic, individually varying emotions are being expressed.

The reference protocol ${ }^{15}$ is divided into two stages: Observational Fear Learning (OFL) and Direct Expression (DE), essentially equivalent to acquisition and test stages in classical fear conditioning, respectively. In OFL, the participant (referred to as the observer) watches a video of another person (referred to as the demonstrator) who undergoes a differential fear conditioning experiment. More precisely, one conditioned stimulus (CS+) is paired

\footnotetext{
${ }^{1}$ Laboratory of Brain Imaging, Nencki Institute of Experimental Biology of Polish Academy of Sciences, 3 Pasteur Str., 02-093 Warsaw, Poland. 'Laboratory of Emotions Neurobiology, BRAINCITY-Centre of Excellence for Neural Plasticity and Brain Disorders, Nencki Institute of Experimental Biology of Polish Academy of Sciences, 3 Pasteur Str., 02-093 Warsaw, Poland. ${ }^{3}$ SWPS University of Social Sciences and Humanities, 10 Kutrzeby Str., 61-719 Poznan, Poland. 'Division of Psychology, Department of Clinical Neuroscience, Karolinska Institutet, Stockholm, Sweden. ${ }^{5}$ These authors contributed equally: Michał Szczepanik and Anna M. Kaźmierowska. ${ }^{\circledR}$ email: e.knapska@nencki.edu.pl
} 
with an electric shock to the forearm of the demonstrator (unconditioned stimulus, US), and the other stimulus (CS-) is not. The demonstrator exhibits strong discomfort in reaction to the shock, which serves as a social US for the observer. In the following DE stage, the observer is told that the same procedure will be applied. However, USs are never actually applied to the observer to guarantee that threat acquisition can occur only through social means. Video recording is made in advance, which allows for precise control over the demonstrator's behavior, but limits the aspect of social involvement and, arguably, ecological validity. Skin conductance responses (SCRs) are recommended as the primary outcome measure. Many elements of this protocol, both with respect to the conditioning procedure and outcome measures are amenable to changes.

Consequently, for the purpose of adapting the protocol, we decided to invite pairs of participants and involve them both in the experimental procedure. Instead of using a prerecorded video of an actor, one of the two participants was asked to become a live demonstrator. Additionally, we decided to recruit pairs of friends expecting that it may increase learning efficiency, which has been shown to be enhanced when the learning model is perceived as similar, for example as he or she belongs to the same social group ${ }^{10,11}$. Moreover, it has been shown that interpersonal liking increases emotional mimicry ${ }^{18}$, which is yet another factor involved in emotional contagion ${ }^{19}$. It has also been suggested that behaviors and emotional expressions of social ingroup members are mimicked preferentially, playing a role in social learning ${ }^{20}$.

In our study, one of the participants (the demonstrator) was subjected to a differential fear conditioning task while being watched by another participant (the observer) through a live video stream. The SCR and fear-potentiated startle (FPS) responses of the observers were recorded as indicators of vicarious fear learning. Although both have been commonly used as measures of aversive conditioning and both typically distinguish between CS+ and CS-, their underlying mechanisms differ; $\operatorname{see}^{21,22}$ for comprehensive reviews. In brief, SCRs are a product of the sudomotor nerves activity and in fear conditioning they most likely reflect a perceived probability of the US. However, SCRs are not specific to threat and can be also elicited by salient or novel stimuli. On the other hand, FPS relies on a simple defensive reflex, in which the primary reflex pathway is modulated by inputs from the amygdala ${ }^{23,24}$, and is typically interpreted in terms of defensive reactivity. Importantly, both measures can be influenced by conscious threat appraisal and reflexive responses to threat. From a methodological standpoint, FPS, unlike SCR, can be probed both during CS and between trials, the latter providing a baseline condition. Using FPS also provides a closer translational link to fear conditioning studies in rodents, where startle methodology is commonly applied and follows similar principles ${ }^{6}$. Moreover, FPS has only recently been applied in the observational fear conditioning design ${ }^{25}$ and no studies from this field have so far employed both these measures, making our study the first to incorporate both.

Our hypotheses were as follows: first, in the OFL phase, we expected an augmented SCR in the observers watching their friends receiving electric shocks. Second, we hypothesized that as a consequence of the pairing between CS+ and social US (friend's reaction to the shock) watched during the learning phase, the observers would develop a conditioned response to the CS+ without directly experiencing the aversive stimulation. This socially driven conditioned reaction was expected to be reflected in stronger SCR and FPS after direct presentation of CS+ compared to CS-, when tested in the DE phase.

\section{Methods}

Participants. 70 male volunteers (35 pairs of friends), aged between 18 and $27(M=21.4, S D=2.2)$ participated in the study. Considering that primary observational fear conditioning effects reported previously, e.g. ${ }^{10}$ were large, our study was sufficiently powered to detect effects of similar size (see the Supplementary Methods for detailed explanation). To be eligible for the study, a pair had to have known each other for at least 3 years (in the recruited group: $M=6.8, S D=4.2$ ) and score sufficiently high (minimum 30 out of 60 points; in the recruited group: $M=50.1, S D=7.3$ ) on the McGill Friendship Questionnaire ${ }^{26}$. All the participants were screened for the ability to recognize the colors used in the task, had no neurological disorders or conditions precluding cutaneous electrical stimulation, did not use psychoactive medication and were not students of psychology or cognitive science. Only heterosexual participants (based on self-declaration) were recruited to restrict the relationships to non-romantic male friendship in order to reduce sample variability. Within each pair, the subjects were randomly assigned roles: one person was the demonstrator (learning model) and the other one was the observer. The protocol of the study was approved by the Ethics Committee of the Faculty of Psychology at the University of Warsaw in accordance with the Declaration of Helsinki. All subjects gave their written informed consents and received financial compensation for participation in the study.

Stimuli and materials. Two large colored squares (blue and yellow) displayed initially on a computer screen in front of the learning model (demonstrator) and later in front of the observer (in OFL and DE stages respectively) served as conditioned stimuli (CS). The assignment of colors to CS+ (squares that might be reinforced with shocks) and CS- (squares that were never reinforced) was counterbalanced across participants. During intertrial intervals, a fixation cross was displayed. The OFL phase consisted of $24 \mathrm{CS}+$ and $24 \mathrm{CS}-$ trials. During the course of the task, 12 CS+ were reinforced with an uncomfortable shock to the ventral part of the upper right forearm of the demonstrator (unconditioned stimulus, US). The CS+/CS- order was pseudo-random with the restriction that any given CS may not be repeated more than twice. The first and last presentation of the CS+ was always reinforced. Two sequences matching these criteria were created and counterbalanced across participants.

In each OFL trial, the CS was presented on the demonstrator's screen for $9 \mathrm{~s}$ and the intertrial interval was randomized between 10 and $15 \mathrm{~s}$. The US, administered in half of the CS+ presentations, started $7.5 \mathrm{~s}$ after CS onset and consisted of five unipolar electrical pulses delivered with $1 \mathrm{~ms}$ duration and a latency of $200 \mathrm{~ms}$ (total stimulation duration $=0.8 \mathrm{~s}$ ). The resulting demonstrator's reaction started straight after the stimulation administration and was likely to co-terminate with the end of the CS+ display. Electrode placement at the upper 

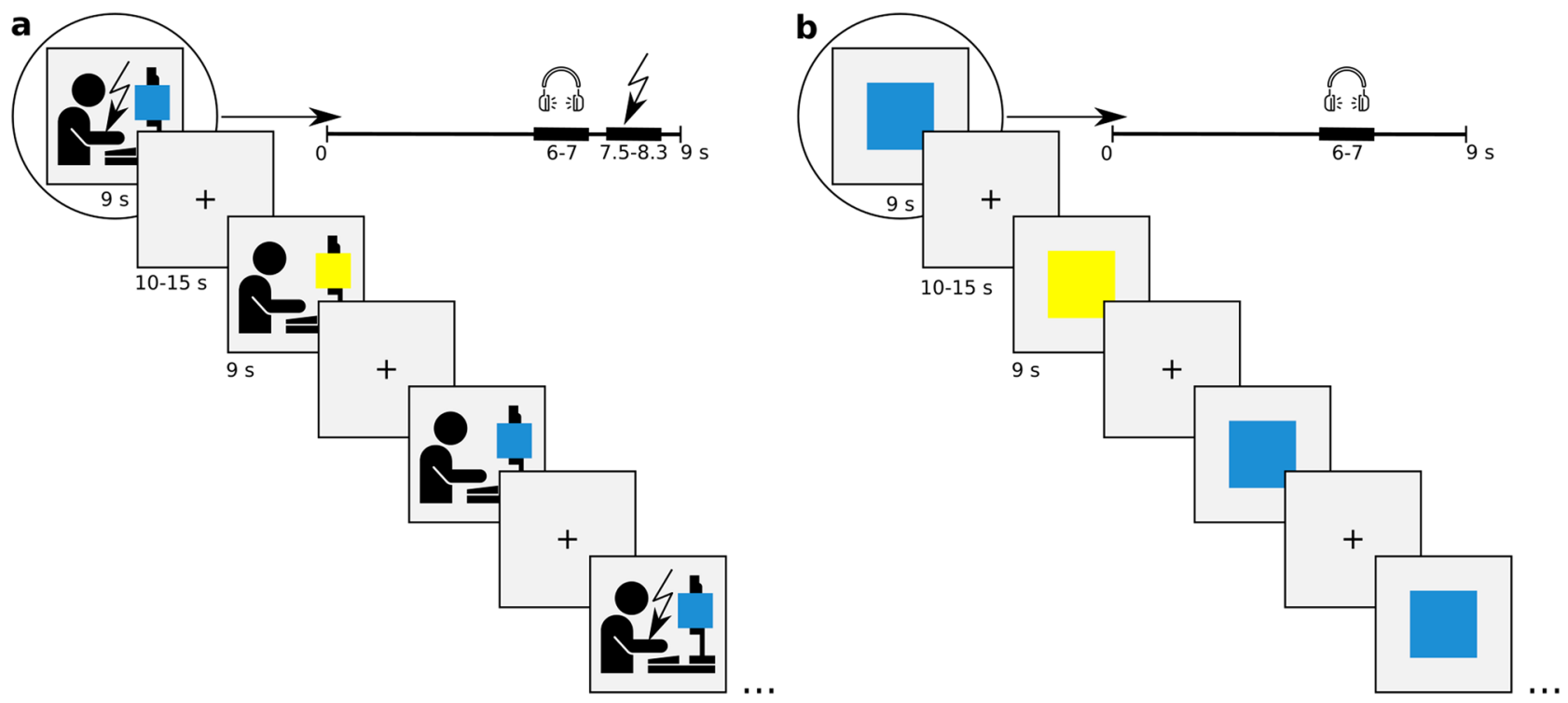

Figure 1. Design of the experiment. (a) In the OFL phase, the observer watched their friend performing a differential fear conditioning task. The conditioned stimuli (blue and yellow squares) were displayed on the demonstrator's screen in a pseudorandom order. Presentation of one of the squares (CS+, here: blue) was accompanied with an uncomfortable electric shock (unconditioned stimulus, US) to the right forearm of the demonstrator with $50 \%$ probability. The other color square served as a stimulus that was never reinforced (CS-, here: yellow). A fixation cross was presented between the stimuli. The observer's SCR and FPS response were used to assess learning. In order to measure the startle reflex magnitude, the white noise burst was pseudorandomly presented through the headphones in half of all types of trials (in which a blue square, yellow square, or fixation cross was presented). (b) In the DE phase, the observer performed the demonstrator's task but the USs were not applied. Instructions provided to the observers did not suggest that the electrical stimulation would accompany only one CS in the OFL phase nor that it would be omitted in the DE phase.

ventral part of the forearm was chosen because in most participants it caused muscle flexion and a resulting hand movement, even at non-painful stimulation intensities. The shock level was adjusted for each demonstrator individually prior to the learning session (see the "Procedure"), so that it was experienced as very uncomfortable but not painful. In order to measure the fear-potentiated startle response, the startle probes were presented to the observer during half of CSs (onsets, randomly chosen, at 6.0, 6.5 or 7.0 s after CS onset) and a quarter of intertrial intervals (onsets between 2.0 and $4.5 \mathrm{~s}$ after fixation cross onset, in order not to interfere with subsequent CS presentations). The acoustic startle probe was a white noise burst ( $80 \mathrm{~dB}(\mathrm{~A})$ and $50 \mathrm{~ms}$ duration) presented binaurally through headphones. Although most studies investigating fear-potentiated reactivity have reported using louder stimuli $(\sim 90 \mathrm{~dB})$, the volume that we used was high enough to elicit startle reflex ${ }^{27}$ and at the same time not overly uncomfortable for the observers (which could interfere with the observational fear acquisition, especially given that the aversive unconditioned stimuli were never direct). The procedure, including number of trials, their timing and the reinforcement ratio were adjusted to match the demands of potential neuroimaging experiments and were based on existing recommendations ${ }^{15}$.

The OFL phase was followed by the DE phase, which consisted of 12 CS +, 12 CS- (displayed on the observer's screen), and no US. Here, timing of CSs, intertrial intervals, stimulus order and startle probe presentations followed the same rules as described above. A scheme presenting the experimental design is shown in Fig. 1.

For observation, an HD-SDI camera connected to a computer screen was used to transmit video (without sound) of one participant to the other. Stimulus presentation was controlled using Presentation v19.0 software (Neurobehavioral Systems, Berkeley, CA, USA). For technical considerations regarding the equipment used, see the Supplementary Methods.

The State-Trait Anxiety Inventory (STAI) $)^{28,29}$ and Basic Empathy Scale for Adults (BES-A) ${ }^{30}$ questionnaires were used to measure state and trait anxiety and empathy. A questionnaire adapted from Ref. ${ }^{31}$ was used for assessment of contingency awareness and a set of Likert-scale based questions were used for assessment of the live demonstration (described in detail in "Procedure" section).

Procedure. We used the experimental protocol of Haaker and colleagues ${ }^{15}$ introducing the following modifications: both demonstrators and observers were invited to the laboratory to take part in the experiment, demonstrators were friends of the subjects, demonstration was transmitted via real time video streaming and fearpotentiated startle was included as an additional measure of conditioning. Upon arrival to the laboratory, the participants were shortly informed about the measurements and stimulation used in the experiment, they filled out the safety form to exclude contraindications for the electrical stimulation and signed the informed consent. Subsequently, they were randomly assigned to their roles as demonstrators and observers and invited to take a seat in one of two adjacent rooms, where they completed the STAI-state questionnaire. Skin conductance, 
electromyography (EMG, used for startle response measurement) and stimulation electrodes were attached and headphones were given to both participants. The demonstrator was seated slightly angled in relation to the computer screen, so that their face, stimulated hand and the computer screen were all visible to the camera relaying the image to the observer's room. Next, both participants were informed about their roles (for detailed instructions see the Supplementary Methods). The demonstrator was told that they would perform a computer task involving colored figures and electric shocks and that their performance would be watched by their friend through the camera. Demonstrators were informed about the color of the figure that would be paired with electrical stimulation. Each demonstrator was also asked to signal each shock occurrence clearly and not try to stifle the discomfort reaction. Besides the natural twitch of the forearm, facial grimace was suggested as an accurate way of demonstrating discomfort. A pre-recorded video presenting an exemplary reaction was shown to each demonstrator in order to provide comparable expressions of discomfort across participants. At the same time, demonstrators were encouraged to behave as naturally as possible and to adjust their reactions so that they would be perceived as reliable. Providing such instructions enabled establishing a compromise between a fully spontaneous demonstrator's behavior (which based on our qualitative observations from extensive pretests was not informative for the observers, as the reactions to non-painful pulses tended to be very subtle) and a fully controllable, carefully prepared videos. After the instructions, shock adjustment was performed. Stimulus intensity was gradually increased and after each trial the participant was asked to describe it using an 8-point scale, ranging from imperceptible (1) to painful (8). The adjustment was stopped when the participant described the shock as 'very unpleasant, but not painful' (6). At the time of the demonstrator's preparations, the observer was seated in a separate room getting habituated to the noise bursts used as startle probes. Subsequently, the observer was informed about their role: they were asked to watch their friend performing a task involving colored figures and electric shocks and to ignore short loud sounds occurring occasionally. They were also told that after observation, they would do the same task themselves.

The OFL phase started with a repeat of the startle habituation (due to interference associated with instructions giving). When the OFL task was over, the video stream was turned off and the demonstrator completed STAI-state for the second time. Immediately after the video stream ended, the observer was informed that it was their turn to do the same task and the DE phase followed. Identical stimulus material and parameters were used, however, no shocks were administered to the subjects.

Finally, the observer filled out an online contingency questionnaire, with questions progressing from openended ("were you able to predict shocks to your friend?" "if yes, how?"), through percentage ratings of the shock occurrence (separately for CS+, CS- and fixation point presented during ITI), to forced choice (between CS+, CS- and fixation point $)^{31}$. Participants were classified as contingency-aware if their forced choice answer was correct and consistent with previous responses (i.e. they either indicated the correct stimulus when asked about presence of a rule or assigned highest shock probability to the correct stimulus). Additionally, the observer rated the demonstrator's performance in terms of the level of discomfort expressed as well as the strength and naturalness of reactions; the observer also rated their level of empathy and degree to which they identified with the demonstrator during the observation. Likert scales ranging from 0 (not at all; very poor) to 9 (very much; very strong) were used for these ratings. Finally, the observer completed STAI-state (for the second time), STAI-trait and BES-a questionnaires. In the end, both participants were debriefed about the study.

Physiological recordings, data scoring and analysis. Skin conductance and startle responses were collected only from the observers and sampled at $2 \mathrm{kHz}$ (for details concerning the signal recording, see the Supplementary Methods).

Skin conductance. The recorded signal was decomposed into tonic and phasic components using cvxEDA ${ }^{32}$. Skin Conductance Responses were scored as the difference between the maximum value occurring between 0 and $6 \mathrm{~s}$ after stimulus onset and the mean value from the preceding $2 \mathrm{~s}$. The response window was limited to $6 \mathrm{~s}$ in order to avoid entanglement of the CS and acoustic startle probe effects. While the phasic component should, by definition, have a zero baseline, the subtraction was done to avoid scoring spontaneous fluctuations occurring before the stimulus. Response window choice was a compromise between the recommendations of the original protocol (foot point of the responses at 0.5-4.5 s after stimulus ${ }^{15}$ ), entire interval response approach ${ }^{33}$ and the constraints imposed by startle application. Amplitude smaller than $0.2 \mu \mathrm{S}$ was treated as no response. Due to timing proximity, responses to the US were measured only for trials containing no startle probes during and after CS presentation, and non-reinforced trials (no US) were also scored, by using an identical time window as when the stimulus was present.

SCR data from subjects for whom less than 5 non-zero responses were observed during the DE phase were excluded from the analysis (leading to exclusion of the SCR data of 3 subjects). The resulting amplitudes were normalized within subjects using a log-transformation: $\log \left(1+\mathrm{SCR} / \mathrm{SCR} \mathrm{max}_{\max }\right)$, where $\mathrm{SCR}_{\max }$ was the highest response found for a given subject. Mean magnitudes (including no-responses as zero amplitude) were calculated for each condition and each participant. Within-subject averages were submitted to group-level analysis.

Fear-potentiated startle. Following the guidelines ${ }^{27}$, the signal was band-pass filtered in the $28-500 \mathrm{~Hz}$ band (4th order Butterworth filter), rectified (i.e. replaced with its absolute value), and smoothed using a $40 \mathrm{~Hz}$ lowpass FIR filter. Each response was scored as the difference between the maximum value in a $20-120 \mathrm{~ms}$ time window and mean value in the -100 to $0 \mathrm{~ms}$ time window (times relative to startle probe onset; trial was scored as 0 if peak amplitude was lower than baseline). Resulting magnitudes were normalized within participants using T-scores ${ }^{27}$. Mean magnitudes were calculated for each participant and each condition and used for grouplevel analysis. 


\begin{tabular}{|l|l|l|l|l|l|}
\hline Assessment of & Question & Min & Max & Me & IQR \\
\hline \multirow{4}{*}{ Demonstrator } & Discomfort expressed & 2 & 8 & 6 & 2 \\
\cline { 2 - 6 } & Strength of reaction & 2 & 8 & 6 & 3 \\
\cline { 2 - 6 } & Naturalness of reaction & 2 & 9 & 8 & 2 \\
\hline \multirow{2}{*}{ Self } & Empathy felt & 0 & 9 & 4 & 4 \\
\cline { 2 - 6 } & Degree of identification & 1 & 9 & 7 & 2 \\
\hline
\end{tabular}

Table 1. Observer's post-experiment rating of the demonstrator's expression as well as their self-empathy (felt during the observation phase) assessment. Me: median, IQR: interquartile range.

All trials were visually inspected for the presence of artifacts, which led to exclusion of data from one participant due to an overall noisy recording; however, no individual trials were discarded. EMG data from two other participants, exhibiting 5 or less non-zero responses throughout the entire experiment, were excluded from the analysis (note that different participants were excluded from the EMG and SCR analyses).

Custom Python scripts utilizing Numpy (https://www.numpy.org/), Scipy ${ }^{34}$, and Bioread (github.com/ uwmadison-chm/bioread) libraries were used for signal preprocessing and scoring of both skin conductance and startle EMG.

For the statistical analyses of psychophysiological responses, repeated measures and mixed-model ANOVAs as well as two-tailed paired $t$ tests were used. The main measures of interest were the conditioned responses (differences between reactions to CS+ and CS-, both skin conductance and startle) examined in the direct expression phase. Ratings based on Likert scales were compared using non-parametric Kruskal-Wallis test. PASW Statistics 18 (SPSS Inc., Chicago, IL, USA) or $\mathrm{R}^{35}$ with $\mathrm{afex}^{36}$, emmeans $\mathrm{em}^{37}$ and $\mathrm{psych}^{38}$ packages were used for statistical analyses.

\section{Results}

Behavioral results. Based on the criteria described above, participants' contingency knowledge was assessed. A total of 14 out of 35 observers were classified as contingency-aware and 21 as contingency-unaware. The non-parametric Kruskal-Wallis test $(N=35)$ revealed no differences in the observers' perception of the demonstrators between contingency-aware $(n=14)$ and -unaware $(n=21)$ groups regarding the 5 studied categories: discomfort expressed, strength of reaction, naturalness of reaction, observer's empathy and observer's degree of identification with the demonstrator (see Table 1).

The summary of remaining psychometric data is included in the Supplementary data and analyses. Most notably, the behavioral measures (including empathy) showed only weak (Pearson's $r$ between -0.33 and 0.26 , median absolute value of $r=0.13$ ) and nonsignificant (without correcting for multiple comparisons) correlation with physiological measures. Additionally, there were no differences between contingency-aware and -unaware participants in scales of empathy and anxiety.

In the group of subjects eligible for SCR analysis, 14 of 32 observers were contingency-aware, while in the group selected for FPS analysis, 13 of 32 observers were contingency-aware.

Skin conductance. In the whole group analysis $(N=32)$, a paired $t$ test revealed a significant difference between reactions measured when observers watched a friend receiving shocks and those recorded during observation of CS+ trials in which no shocks were applied, $t(31)=8.37, p<0.001, d=1.69$ (see Fig. 2a). Regarding the SCRs to colored squares presented throughout the experiment, in a repeated-measures ANOVA (with stimulus type and task phase as within-subject factors) we observed no differential reactions to CS+ compared to CS- presentation either in the OFL or in the DE phase and no main effects were found.

Having obtained a low ratio of the CS+/US contingency, we decided to check whether the contingency knowledge moderates the relationship between SCRs and the conditioned stimuli (CS+ and CS-). For this purpose, we used a mixed model ANOVA with the skin conductance response as a dependent variable, stimulus type as a within-subject factor, and contingency knowledge as a between-subject factor. In the direct expression phase we found the stimulus (CS+ vs. CS - ) $\times$ contingency knowledge (known vs. unknown) interaction, $F(1,30)=12.29$, $p=0.001, \eta_{\mathrm{p}}{ }^{2}=0.29$. Post hoc comparisons (with Bonferroni correction) revealed a stronger skin conductance response to CS+ $(M=0.11, S D=0.05)$ as compared to CS- $(M=0.07, S D=0.04, p=0.002, d=0.88,95 \% \mathrm{CI}$ $[0.02,0.07])$ only in participants who indicated the CS+/US contingency correctly. Moreover, skin conductance response to $C S+$ was stronger in the group of contingency-aware $(M=0.11, S D=0.05)$ compared to -unaware participants $(M=0.07, S D=0.05, p=0.015, d=0.80,95 \%$ CI [0.01, 0.08]; see Fig. 2b). Analogous analysis, using only early direct expression trials is reported in Supplementary data and analyses.

Fear-potentiated startle. In the whole group analysis $(N=32)$, in order to check for the differences in FPS responses to different stimuli depending on the phase of the experiment, we ran a repeated measures ANOVA with the startle response as a dependent variable and stimulus type and task phase as within-subject factors. We found the main effect of phase, $F(1,31)=81.87, p<0.001, \eta_{\mathrm{p}}{ }^{2}=0.73$. Post hoc comparisons (with Bonferroni correction) revealed a stronger FPS response in the $\mathrm{DE}(M=54.14, S D=2.59)$ compared to the OFL phase $(M=47.93, S D=1.30, p<0.001, d=3.03,95 \%$ CI $[4.81,7.62]$; see Fig. 3a). 
a

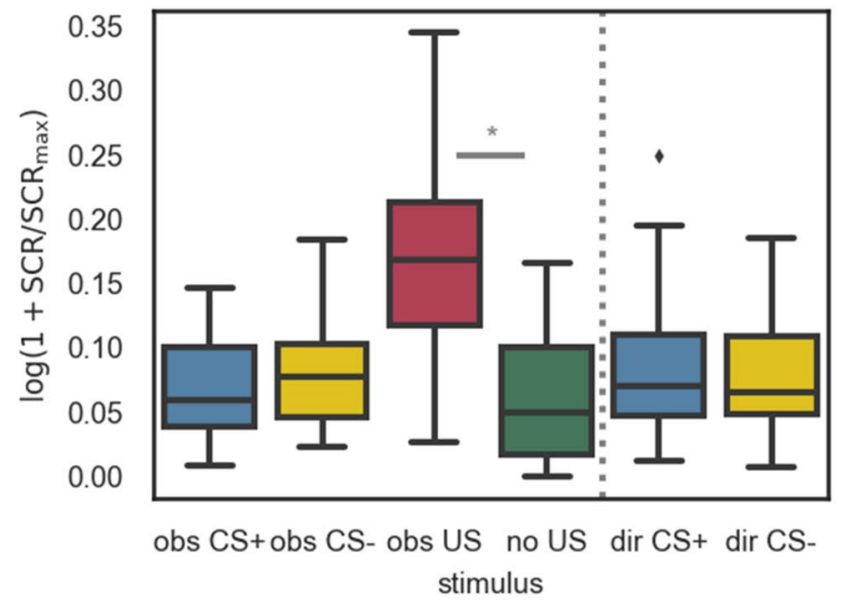

b

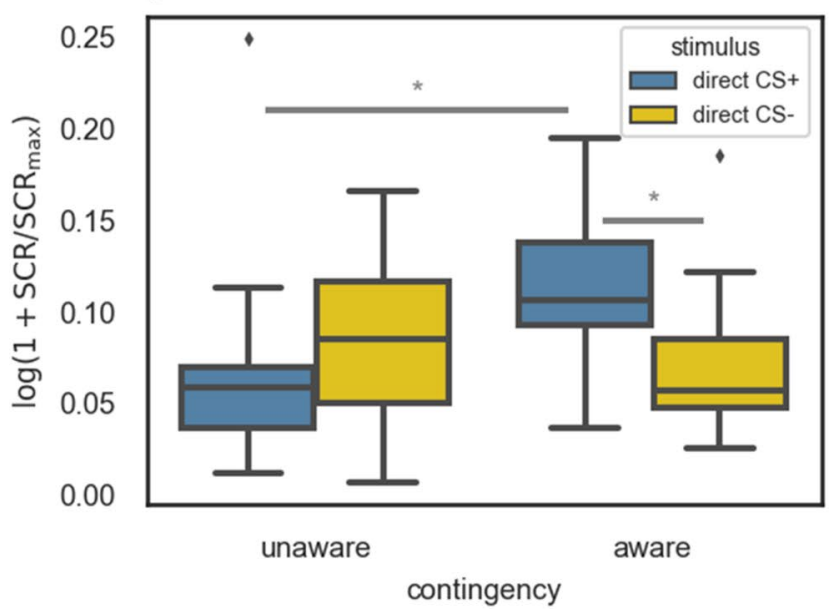

Figure 2. (a) SCR magnitudes to stimuli presented in the experiment: obs CS+ and obs CS- (appearance of CS+/CS - in the OFL phase), obs US and no US (observation of a friend receiving/not receiving electric shock during CS+ in the OFL phase), dir CS+ and dir CS- (appearance of CS+/CS- in the DE phase). Substantial differences are marked. $N=32$. (b) SCR magnitudes to the appearance of the CS+ and CS - in the DE phase of the experiment for contingency-aware $(n=14)$ and -unaware $(n=18)$ participants separately. The stimulus (CS+ vs. CS - ) $\times$ contingency (aware vs. unaware) interaction was found, $F(1,30)=12.29, p=0.001, \eta_{\mathrm{p}}{ }^{2}=0.29$, and substantial effects of the post-hoc comparisons are marked. Error bars indicate $1.5^{\star}$ IQR beyond the 1st quartile and above the $3 \mathrm{rd}$ quartile. The ${ }^{\star}$ symbol was used for $p<0.05$.

a

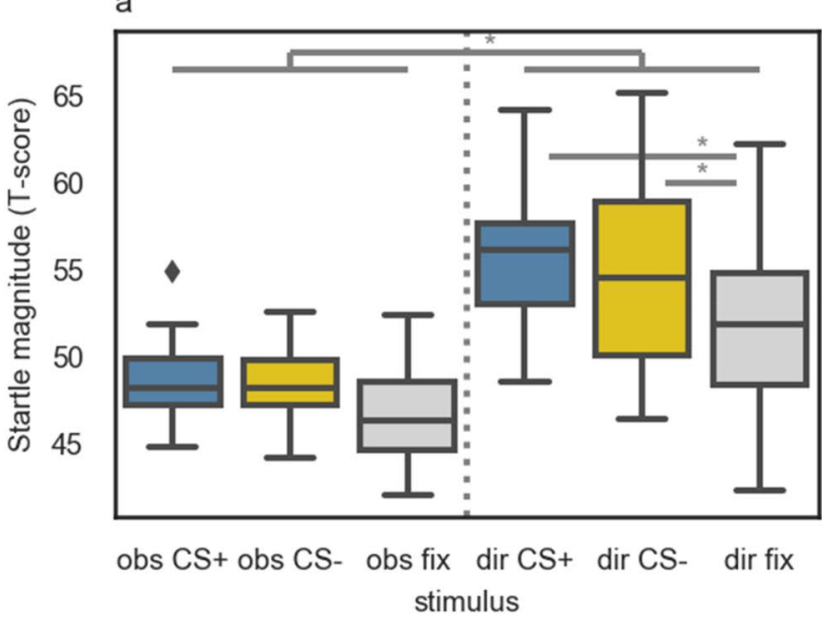

b

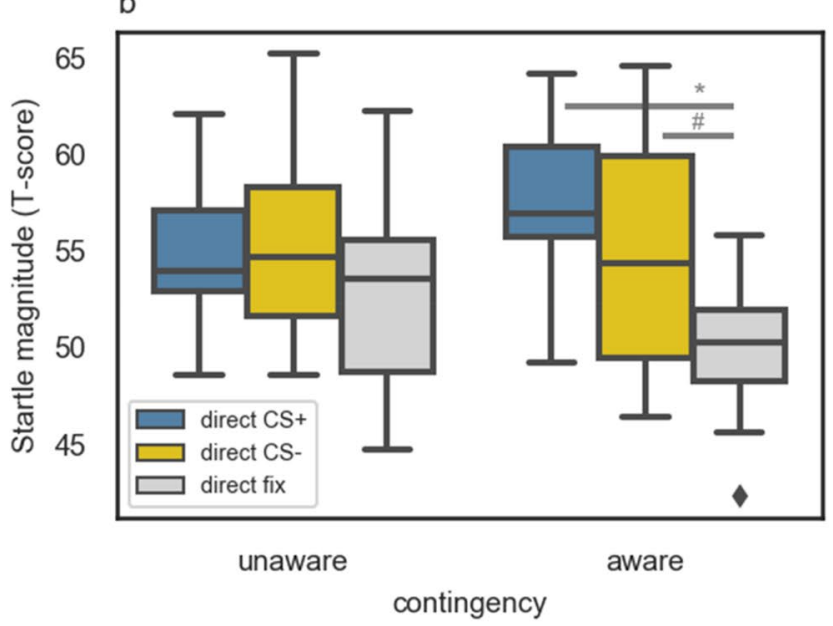

Figure 3. (a) FPS responses measured during different stimuli presented in the experiment: obs CS+, obs CS- and obs fix (presentation of CS+/CS-/fixation cross in the OFL phase), dir CS+, dir CS- and dir fix (presentation of CS+/CS-/fixation cross in the DE phase). Substantial differences are marked. $N=32$. (b) FPS magnitudes measured in the DE phase: results of contingency-aware $(n=13)$ and -unaware $(n=19)$ participants are grouped separately. A trend toward the stimulus (CS+ vs. CS - vs. fixation cross) $\times$ contingency (aware vs. unaware) interaction was found, $F(2,60)=2.59, p=0.083, \eta_{\mathrm{p}}{ }^{2}=0.08$, and substantial effects of the post-hoc comparisons are marked. Error bars indicate $1.5^{\star} \mathrm{IQR}$ beyond the 1 st quartile and above the 3 rd quartile. The * symbol was used for $p<0.05$, and \# symbol for $p<0.1$.

To check whether there was an effect of contingency knowledge on FPS responses to different stimuli in the DE phase, we performed a mixed model ANOVA analogous to the analysis of the SCR data. We found a significant main effect of stimulus (CS+ vs. CS- vs. fixation cross), $F(2,60)=8.16, p=0.001, \eta_{\mathrm{p}}{ }^{2}=0.21$. Further post hoc comparisons (with Bonferroni correction) including all subjects showed that the FPS reaction to both CS+ $(M=55.71, S D=4.08)$ and $C S-(M=54.99, S D=5.21)$ was stronger than the one measured during the fixation cross presentation $(M=51.73, S D=4.55)$; for CS $+>$ fixation cross: $p<0.001, d=0.92,95 \%$ CI $[1.97,6.99]$; for CS- > fixation cross: $p=0.034, d=0.67,95 \%$ CI $[0.22,6.85]$; see Fig. 3a. There was also a trend toward the stimulus (CS+ vs. CS - vs. fixation cross) $\times$ contingency knowledge (aware vs. unaware) interaction, $F(2,60)=2.59$, $p=0.083, \eta_{\mathrm{p}}{ }^{2}=0.08$. Results of the post hoc comparisons (with Bonferroni correction) revealed that only in the group of contingency-aware participants, the response to both CS+ $(M=57.13, S D=4.56)$ and CS- $(M=55.01$, 
$S D=6.17)$ was stronger than the response to a fixation cross $(M=49.10, S D=3.85)$; for $C S+>$ fixation cross: $p<0.001, d=1.69,95 \%$ CI $[3.27,11.00]$; for CS $->$ fixation cross: $p=0.056, d=0.97,95 \%$ CI $[-0.10,10.12]$; see Fig. $3 \mathrm{~b}$. This trend suggests that the effect of the potentiated startle response to both conditioned stimuli might be specific to only the contingency-aware participants. Analogous analysis, using only early direct expression trials is reported in Supplementary data and analyses.

\section{Discussion}

In the present study the observational fear conditioning was tested in a paradigm with improved ecological validity. Most previous studies on this phenomenon have used prepared video recordings with actors unknown to the observers. Instead, we arranged a real-time experiment involving participants who were significant to each other. Further, to better reflect activity of the defensive system, in addition to SCR, we used FPS. As the latter is commonly used in rodent studies, it additionally allows for across species comparisons. Both measures confirmed that acquisition of fear through observation was successful. They also showed that differential fear learning was dependent on declarative knowledge of the CS+/US contingency.

We were particularly interested in the results obtained during the direct expression (DE) phase, as they reflected the observational fear learning efficiency. These results confirmed that the observers learned to respond with physiologically stronger reactions to threat, but only when they were aware of the CS-US contingency. Specifically, the contingency-aware participants responded with stronger SCRs to the direct CS+ (vs. CS-) and increased startle potentiation towards both CSs (vs. fixation cross). The latter effect suggests that the aversive learning generalized; indeed, it is possible that during the OFL phase participants learned to associate aversive feelings with both squares, as opposed to the fixation cross, and, as a consequence, during the DE phase, both CSs (and not the fixation cross) elicited augmented startle responses.

When taking a closer look at the SCR results, two effects are apparent. First, observational US elicited stronger and more robust reactions than any of the conditioned stimuli in the DE phase. This means that the US was perceived the way we expected-which is important, considering that observing the aversive qualities of the US is a prerequisite for threat learning. Second, differential conditioning of the SCRs was observed only in the contingency-aware participants. This observation could be made because the low rate of contingency awareness declared in the post-experimental questionnaire allowed splitting the participants into two groups; it suggests that learning about CS-US contingency can be separated from the response to the observational US.

In the case of FPS results, we observe several effects. Overall, the reactions were stronger in the DE compared to the OFL stage, suggesting that an elevated sense of threat was induced during this part of the experiment, and that following observation the observers indeed expected to experience the aversive stimulation themselves. This remains in line with the interpretation of startle response as reflecting defensive preparation or reactivity to stimuli suggesting direct threat. Furthermore, in the DE stage, startle probes presented during both conditioned stimuli elicited heightened responses compared to the ones measured during intertrial intervals, suggesting that the acquired fear was generalized (nonspecific); although this effect could also be attributed partially to the fact that both CSs were more salient stimuli than the fixation point presented during ITI. We observed a trendlevel interaction with contingency knowledge, which upon inspection suggests that the effect is driven by the contingency-aware participants. Although the SCR and FPS results did not show one-to-one correspondence, the information they provide is complementary and consistent. It seems that both attention and defensive reactivity are increased as a consequence of observational fear conditioning in the tested paradigm and that these effects are specific for participants reporting declarative knowledge about the CS+/US relationship.

The physiological results replicate the findings of previous works which manipulated various elements in a video-based vicarious learning procedure and consistently showed differential conditioning effects measured by $\mathrm{SCR}^{10-12}$ and $\mathrm{FPS}^{25}$. Interestingly, in the aforementioned studies, an overwhelming majority of the participants correctly identified the CS+/US contingency and the few who did not were excluded from analyses ${ }^{39}$. In our experiment, the general learning efficiency (declarative contingency knowledge) was low, with 14 out of 35 participants correctly identifying the relationship between CS and US. Therefore, the largest difference observed in the current results lies in the low ratio of the CS+/US contingency observed.

The changes we introduced to the reference protoco ${ }^{15}$ were designed to increase its ecological validity. To improve the immediacy and realism of the threat learning situation, we invited both demonstrator and observer (who were well known to each other) to the laboratory and carried out the observational stage via live streaming. At the same time, the increase of realism came at a cost of lessening our control over experimental conditions: although the demonstrators received general instructions about how they should react to the unpleasant stimulus, each of them still reacted in their own individual way. Such reactions could not be influenced once the experiment started and were not selected through video editing before the experiment ${ }^{15}$. While this improved realism, it might have also increased ambiguity (leading to lowered rate of contingency awareness), since spontaneous displays of emotions are smaller in amplitude and have different internal timing than posed emotional expressions ${ }^{18}$. The ambiguity, however, should have been reduced by the fact that the observers were familiar with the demonstrators and thus more likely to be sensitive to their emotional expressions. While our ecologicallyaimed modifications likely contributed to the low contingency knowledge, other factors might come into play as well. First, the inclusion of startle probes ${ }^{40}$ and low (50\%) CS + reinforcement ratio might have further contributed to the creation of a weak learning situation. It is possible that auditory stimuli not only disrupted participants' attention but also introduced an additional aversive component which interfered with the clear CS+/US association. Increase of the CS+ reinforcement ratio should be considered as a possibility for the contingency learning enhancement. Furthermore, the demonstrators were seated in an ordinary room, rather than against a clear and contrasting background, which might have introduced additional visual distractors. Finally, in our instructions we informed the observers that the demonstrator would experience visual stimuli and electric shocks, but did 
not suggest that any relation between the two might exist. This was in accordance with the reference protocol ${ }^{15}$, but in contrast with some previous studies ${ }^{14,41}$ where instructions were more explicit.

We find the low level of contingency knowledge and its impact on conditioning measures interesting (as it highlights the role of the observer's attention and the observational stimulus quality), but not surprising, given that the role of contingency knowledge has been described for various conditioning protocols and conditioning measures, e.g. ${ }^{31,42,43}$. Skin conductance response has been reported as a measure reflecting contingency learning ${ }^{44}$, while potentiation of the acoustic startle reflex has been claimed to be more valence-specific and less dependent on attention ${ }^{45}$, although see ${ }^{46}$, but its relation to contingency awareness is still unclear. We showed that in a real time procedure, contingency learning is reflected in augmented physiological reactions to potential threat, as indicated by stronger SCR and FPS responses of the contingency-aware observers. Thanks to employing two different psychophysiological measures of conditioning, we demonstrated two effects: differentiation of the SCR and generalization of the FPS response, both relying on contingency knowledge. Our findings indicate an important characteristic of the human defensive system, suggesting its high sensitivity to all the cues related to the potential threat as well as its dependence on conscious processing of the context.

It is important to emphasize the significance of this study for further development of the vicarious fear learning research. The design proposed here is conceptually similar to the direct threat models of observational fear conditioning in rodents ${ }^{47,48}$, in which two interacting animals are typically used. The main advantage of our design is the real involvement of both demonstrator and observer as well as close relationship between both participants (in rodent studies, subjects are typically cage mates). Furthermore, acoustic startle response is commonly measured in rodent studies and its underlying neural circuit, involving the brainstem and centromedial amygdala, is conserved across species ${ }^{23,49}$. These commonalities give rise to a whole range of possibilities for studying mechanisms of social fear acquisition across species. Research questions that might be answered owing to translational studies involve, for example, comparison of neural circuits related to observational fear learning in humans and rodents, investigation of the impact of participants' familiarity on social fear learning (on both behavioral and neural level), and examination of different channels used for fear transmission (e.g. odor communication in humans).

One of the main limitations of the current study is a relatively small sample size, especially when group comparisons are considered. Although the previous reports suggested that the primary effects are strong and that reliable results should be observed also for current size subgroups, the interaction effects we observed are small. Nevertheless, our results are consistent with previous findings and validate the methodological frame for further ecological studies.

Arguably, the interaction between two participants could have been more natural if they were in the same room throughout the experiment. However, considering that the participants started the experiment together, we believe that their sense of involvement was maintained and likely much higher than in the case of recorded actors. At the same time, video streaming is more viable for different applications, such as fMRI or eye tracking. Thus, the proposed design constitutes a trade-off between naturalistic conditions and methodological requirements of potential applications. Finally, one aspect which the current study left unaddressed was the effects of specific dyads, for example due to relationship strength ${ }^{50-52}$, on observational learning. We believe it might be addressed more accurately by further research, with larger and more varied groups of participants.

To conclude, in this work we show that participants watching their friends acquiring fear in real time respond strongly to the friend's reactions to shock and can learn about the observed threat vicariously, although the learning efficiency is lower than reported in previous studies. The results suggest that the process of learning fear through observation may be composed of two separable components: an automatic, non-specific emotional reaction to the response of the demonstrator (which can serve as social unconditioned stimulus, US) and learning about predicting stimulus (CS)-US contingency. Further studies are needed to describe the factors underlying successful observational fear learning under ecological conditions.

\section{Data availability}

Data from psychophysiological recordings are stored in an OSF repository and are available at https://osf.io/ $\mathrm{d} 3 \mathrm{wxn}$ [note: this is a view only link for review, OSF project will be made public upon publication]. Code replicating analyses reported here is available at https://github.com/mslw/vic-fear-learning-physio.

Received: 11 May 2020; Accepted: 7 September 2020

Published online: 12 October 2020

\section{References}

1. Preston, S. D. \& de Waal, F. B. M. Empathy: its ultimate and proximate bases. Behav. Brain Sci. 25, 1-20 (2002).

2. de Waal, F. B. M. The 'Russian Doll' model of empathy and imitation: from mirror neurons to empathy. In On Being Moved Vol. 68 (ed. Bråten, S.) 49-69 (John Benjamins Publishing Company, Amsterdam, 2007).

3. Huber, A., Barber, A. L. A., Faragó, T., Müller, C. A. \& Huber, L. Investigating emotional contagion in dogs (Canis familiaris) to emotional sounds of humans and conspecifics. Anim. Cogn. 20, 703-715 (2017).

4. Adriaense, J. E. C., Martin, J. S., Schiestl, M., Lamm, C. \& Bugnyar, T. Negative emotional contagion and cognitive bias in common ravens (Corvus corax). Proc. Natl. Acad. Sci. U.S.A. 116, 11547-11552 (2019).

5. Mineka, S., Davidson, M., Cook, M. \& Keir, R. Observational conditioning of snake fear in rhesus monkeys. J. Abnorm. Psychol. 93, 355-372 (1984).

6. Knapska, E. et al. Between-subject transfer of emotional information evokes specific pattern of amygdala activation. Proc. Natl. Acad. Sci. U.S.A. 103, 3858-3862 (2006).

7. Knapska, E., Mikosz, M., Werka, T. \& Maren, S. Social modulation of learning in rats. Learn. Mem. 17, 35-42 (2010).

8. Lindström, B., Haaker, J. \& Olsson, A. A common neural network differentially mediates direct and social fear learning. Neuroimage 167, 121-129 (2018). 
9. Olsson, A. \& Phelps, E. A. Learned fear of 'unseen' faces after Pavlovian, observational, and instructed fear. Psychol. Sci. 15, 822-828 (2004).

10. Golkar, A. \& Olsson, A. The interplay of social group biases in social threat learning. Sci. Rep. 7, 7685 (2017).

11. Golkar, A., Castro, V. \& Olsson, A. Social learning of fear and safety is determined by the demonstrator's racial group. Biol. Lett. 11, 20140817 (2015).

12. Olsson, A. et al. Vicarious fear learning depends on empathic appraisals and trait empathy. Psychol. Sci. 27, 25-33 (2016).

13. Williams, A. L. \& Conway, C. C. Empathy does not amplify vicarious threat learning. Behav. Res. Ther. 127, 103577 (2020).

14. Pärnamets, P., Espinosa, L. \& Olsson, A. Physiological synchrony predicts observational threat learning in humans. Proc. Biol. Sci. 287, $20192779(2020)$.

15. Haaker, J., Golkar, A., Selbing, I. \& Olsson, A. Assessment of social transmission of threats in humans using observational fear conditioning. Nat. Protoc. 12, 1378-1386 (2017).

16. Bottenhorn, K. L. et al. Cooperating yet distinct brain networks engaged during naturalistic paradigms: a meta-analysis of functional MRI results. Netw. Neurosci. 3, 27-48 (2019).

17. Matusz, P. J., Dikker, S., Huth, A. G. \& Perrodin, C. Are we ready for real-world neuroscience?. J. Cogn. Neurosci. 31, 327-338 (2019).

18. McIntosh, D. Sponteous facial mimicry, liking and emotional contagion. Polish Psychol. Bull. 1, 31-42 (2006).

19. Olszanowski, M., Wróbel, M. \& Hess, U. Mimicking and sharing emotions: a re-examination of the link between facial mimicry and emotional contagion. Cogn. Emot. 34, 367-376 (2020).

20. Kavanagh, L. C. \& Winkielman, P. The functionality of spontaneous mimicry and its influences on affiliation: an implicit socialization account. Front. Psychol. https://doi.org/10.3389/fpsyg.2016.00458 (2016).

21. Lonsdorf, T. B. et al. Don't fear 'fear conditioning': methodological considerations for the design and analysis of studies on human fear acquisition, extinction, and return of fear. Neurosci. Biobehav. Rev. 77, 247-285 (2017).

22. Ojala, K. E. \& Bach, D. R. Measuring learning in human classical threat conditioning: translational, cognitive and methodological considerations. Neurosci. Biobehav. Rev. 114, 96-112 (2020).

23. Koch, M. The neurobiology of startle. Prog. Neurobiol. 59, 107-128 (1999).

24. Davis, M. Neural systems involved in fear and anxiety measured with fear-potentiated startle. Am. Psychol. 61, 741-756 (2006).

25. Selbing, I. \& Olsson, A. Anxious behaviour in a demonstrator affects observational learning. Sci. Rep. 9, 9181 (2019).

26. Mendelson, M. J. \& Aboud, F. E. Measuring friendship quality in late adolescents and young adults: McGill Friendship Questionnaires. Can. J. Behav. Sci. 31, 130 (1999).

27. Blumenthal, T. D. et al. Committee report: guidelines for human startle eyeblink electromyographic studies. Psychophysiology 42, $1-15$ (2005).

28. Spielberger, C. D., Gorsuch, R. L., Lushene, R., Vagg, P. R. \& Jacobs, G. A. Manual for the State-Trait Anxiety Inventory (Consulting Psychologists Press, San Jose, 1983).

29. Spielberger, C., Strelau, J., Tysarczyk, M. \& Wrześniewski, K. STAI-Inwentarz Stanu i Cechy Lęku (Pracownia Testów Psychologicznych Polskiego Towarzystwa Psychologicznego, Warsaw, 2012).

30. Carré, A., Stefaniak, N., D’Ambrosio, F., Bensalah, L. \& Besche-Richard, C. The Basic Empathy Scale in adults (BES-A): factor structure of a revised form. Psychol. Assess. 25, 679-691 (2013).

31. Weidemann, G., Satkunarajah, M. \& Lovibond, P. F. I Think, therefore eyeblink: the importance of contingency awareness in conditioning. Psychol. Sci. 27, 467-475 (2016).

32. Greco, A., Valenza, G., Lanata, A., Scilingo, E. P. \& Citi, L. cvxEDA: A convex optimization approach to electrodermal activity processing. IEEE Trans. Biomed. Eng. 63, 797-804 (2016).

33. Pineles, S. L., Orr, M. R. \& Orr, S. P. An alternative scoring method for skin conductance responding in a differential fear conditioning paradigm with a long-duration conditioned stimulus. Psychophysiology 46, 984-995 (2009).

34. Virtanen, P. et al. scipy/scipy: SciPy 1.1.0. https://doi.org/10.5281/zenodo.1241501 (2018).

35. R Core Team. R: A Language and Environment for Statistical Computing. https://www.R-project.org/ (2020).

36. Singmann, H., Bolker, B., Westfall, J., Aust, F. \& Ben-Shachar, M. S. afex: Analysis of Factorial Experiments. https://CRAN.R-proje ct.org/package $=$ afex $(2020)$.

37. Lenth, R. emmeans: Estimated Marginal Means, aka Least-Squares Means. https://CRAN.R-project.org/package=emmeans (2020).

38. Revelle, W. psych: Procedures for Psychological, Psychometric, and Personality Research. https://CRAN.R-project.org/package=psych (2020).

39. Golkar, A. \& Olsson, A. Immunization against social fear learning. J. Exp. Psychol. Gen. 145, 665-671 (2016).

40. Sjouwerman, R., Niehaus, J., Kuhn, M. \& Lonsdorf, T. B. Don't startle me-Interference of startle probe presentations and intermittent ratings with fear acquisition. Psychophysiology 53, 1889-1899 (2016).

41. Olsson, A., Nearing, K. I. \& Phelps, E. A. Learning fears by observing others: the neural systems of social fear transmission. Soc. Cogn. Affect. Neurosci. 2, 3-11 (2007).

42. Baeuchl, C., Hoppstädter, M., Meyer, P. \& Flor, H. Contingency awareness as a prerequisite for differential contextual fear conditioning. Cogn. Affect. Behav. Neurosci. 19, 811-828 (2019).

43. Tabbert, K. et al. Influence of contingency awareness on neural, electrodermal and evaluative responses during fear conditioning. Soc. Cogn. Affect. Neurosci. 6, 495-506 (2011).

44. Sevenster, D., Beckers, T. \& Kindt, M. Fear conditioning of SCR but not the startle reflex requires conscious discrimination of threat and safety. Front. Behav. Neurosci. 8, 32 (2014).

45. Lang, P. J., Bradley, M. M. \& Cuthbert, B. N. Emotion, attention, and the startle reflex. Psychol. Rev. 97, 377-395 (1990).

46. Mertens, G. \& De Houwer, J. Potentiation of the startle reflex is in line with contingency reversal instructions rather than the conditioning history. Biol. Psychol. 113, 91-99 (2016).

47. Jeon, D. et al. Observational fear learning involves affective pain system and Cav1.2 $\mathrm{Ca}^{2+}$ channels in ACC. Nat. Neurosci. 13, $482-488$ (2010).

48. Jeon, D. \& Shin, H.-S. A mouse model for observational fear learning and the empathetic response. Curr. Protoc. Neurosci. Chapter 8, Unit 8.27 (2011).

49. Kuhn, M. et al. The neurofunctional basis of affective startle modulation in humans: evidence from combined facial electromyography and functional magnetic resonance imaging. Biol. Psychiatry 87, 548-558 (2020).

50. de Vries, B. The understanding of friendship: an adult life course perspective, chapter 14. In Handbook of Emotion, Adult Development (eds Magai, C. \& McFadden, S. H.) 249-268 (Academic Press, Cambridge, 1996).

51. Bahns, A. J., Crandall, C. S., Gillath, O. \& Preacher, K. J. Similarity in relationships as niche construction: choice, stability, and influence within dyads in a free choice environment. J. Pers. Soc. Psychol. 112, 329-355 (2017).

52. Hall, J. A. How many hours does it take to make a friend?. J. Soc. Pers. Relat. 36, 1278-1296 (2019).

\section{Acknowledgements}

We would like to thank Artur Marchewka for his valuable comments regarding the study design and the interpretation of results. Data collection and analysis were sponsored by National Science Centre grant 2015/19/B/ HS6/02209. Ewelina Knapska was supported by European Research Council Starting Grant (H 415148). 


\section{Author contributions}

All the authors participated in designing the experiment, M.S. and A.K. conducted the experiment, performed the data analysis and drafted the manuscript, and J.M.M., M.W., A.O. and E.K. provided revisions. All authors approved the final version of the manuscript for submission.

\section{Competing interests}

The authors declare no competing interests.

\section{Additional information}

Supplementary information is available for this paper at https://doi.org/10.1038/s41598-020-74113-W.

Correspondence and requests for materials should be addressed to E.K.

Reprints and permissions information is available at www.nature.com/reprints.

Publisher's note Springer Nature remains neutral with regard to jurisdictional claims in published maps and institutional affiliations.

(c) (i) Open Access This article is licensed under a Creative Commons Attribution 4.0 International License, which permits use, sharing, adaptation, distribution and reproduction in any medium or format, as long as you give appropriate credit to the original author(s) and the source, provide a link to the Creative Commons licence, and indicate if changes were made. The images or other third party material in this article are included in the article's Creative Commons licence, unless indicated otherwise in a credit line to the material. If material is not included in the article's Creative Commons licence and your intended use is not permitted by statutory regulation or exceeds the permitted use, you will need to obtain permission directly from the copyright holder. To view a copy of this licence, visit http://creativecommons.org/licenses/by/4.0/.

(C) The Author(s) 2020 